\title{
Monitoring perception reliability in autonomous driving: Distributional shift detection for estimating the impact of input data on prediction accuracy
}

\author{
Sakshi Goyal \\ STTech GmbH \\ Munich, Germany \\ sakshi.goyal@sttech.de \\ Tetiana Lytvynenko \\ STTech GmbH \\ Munich, Germany \\ tetiana.lytvynenko@sttech.de
}

\author{
Franz Hell \\ STTech GmbH \\ Munich, Germany \\ franz.hell@sttech.de \\ Feng Liu \\ TTE-RAMS Lab, Huawei Munich \\ Research Center \\ Munich, Germany \\ feng.liu@huawei.com
}

\author{
Gereon Hinz \\ STTech $\mathrm{GmbH}$ \\ Munich, Germany \\ gereon.hinz@sttech.de \\ Chen Yiqiang \\ RAMS Lab, Huawei Technologies Co. \\ Ltd. , P. R. \\ China \\ chenyiqiang@huawei.com
}

Ke Pei

TTE-RAMS Lab, Huawei Munich

Research Center

Munich, Germany

peike@huawei.com

\author{
Alois Knoll \\ Technical University of Munich \\ Munich, Germany \\ knoll@in.tum.de
}

\begin{abstract}
Deep neural networks are at the heart of safety-critical applications such as autonomous driving. Distributional shift is a typical problem in predictive modeling, when the feature distribution of inputs and outputs varies between the training and test stages. When used on data different from the training distribution, neural networks provide little or no performance guarantees on such out-of-distribution (OOD) inputs. Monitoring distributional shift can help assess reliability of neural network predictions with the purpose of predicting potential safety-critical contexts. With our research, we evaluate state of the art OOD detection methods on autonomous driving camera data, while also demonstrating the influence of OOD data on the prediction reliability of neural networks. We evaluate three different OOD detection methods: As a baseline method we employ a variational autoencoder (VAE) trained on the similar data as the perception network (depth estimation) and use a reconstruction error based out of distribution measure. As a second approach, we choose to evaluate a method termed Likelihood Regret, which has been shown to be an efficient likelihood based OOD measure for VAEs. As a third approach, we evaluate another recently introduced method based on generative modelling termed SSD, which uses self-supervised representation learning followed by a distance based detection in the feature space, to calculate the outlier score. We compare all 3 methods and evaluate them concurrently with the error of an depth estimation network. Results show
\end{abstract}

This work is licensed under a Creative Commons Attribution International 4.0 License.

CSCS '21, November 30, 2021, Ingolstadt, Germany

(C) 2021 Copyright held by the owner/author(s)

ACM ISBN 978-1-4503-9139-9/21/11.

https://doi.org/10.1145/3488904.3493382 that while the reconstruction error based OOD metric is not able to differentiate between in and out of distribution data across all scenarios, the likelihood regret based OOD metric as well as the SSD outlier score perform fairly well in OOD detection. Their metrics are also highly correlated with perception error, rendering them promising candidates for an autonomous driving system reliability monitor.

\section{CCS CONCEPTS}

- Computing methodologies $\rightarrow$ Scene anomaly detection.

\section{KEYWORDS}

ood detection, distributional shift, reliability, autonomous driving, camera, image

\section{ACM Reference Format:}

Sakshi Goyal, Franz Hell, Gereon Hinz, Tetiana Lytvynenko, Feng Liu, Chen Yiqiang, Ke Pei, and Alois Knoll. 2021. Monitoring perception reliability in autonomous driving: Distributional shift detection for estimating the impact of input data on prediction accuracy. In Computer Science in Cars Symposium (CSCS '21), November 30, 2021, Ingolstadt, Germany. ACM, New York, NY, USA, 9 pages. https://doi.org/10.1145/3488904.3493382

\section{INTRODUCTION}

Distributional shift is a typical problem in predictive modeling, when the distribution of inputs and outputs varies between the training and test stages. For causes ranging from experimental design bias to the irreproducibility of testing conditions at training time, dataset shift is evident in most practical applications [Joaquin et al. 2008]. Deep neural networks are at the core of a slew of safetycritical applications, from autonomous driving [Ramanagopal et al. 2018] to biometric authentication [Gunther et al. 2017; Masi et al. 2019]. When used on data different from the training distribution, 
neural networks provide little or no performance guarantees on such out-of-distribution (OOD) inputs. The prediction may not only be incorrect, but also confidently so, posing a risk to model deployment in real-world applications [Joaquin et al. 2008; Volpi and Murino 2019].

A neural network component's reliability can be assessed using either white-box or black-box approaches. White-box approaches track the internal behavior of a neural network component and necessitate network access as well as extensive domain knowledge in order to create nontrivial models that approximate the network's uncertainty. On the other hand, black-box approaches can help estimate the networks uncertainty by monitoring the relationship between the current input data and the input images used during training [Stocco et al. 2020]. Take, for example, an object detection model that has solely been trained on sunny images of suburban regions. If images representing a narrow urban street during bad weather are presented to the detector, the model will still output detections, but also likely will miss some from time to time. Ideally we would like to warn the autonomous driving system of a potential drop in the reliability of these detections, as neural networks are known to perform worse in unknown contexts [Joaquin et al. 2008; Volpi and Murino 2019]. In this paper, we focus on black-box reliability estimation. Black-box confidence measures have the benefit of being independent of the neural network designs used for various perceptual tasks such as object detection or depth estimation. Monitoring distributional shift can help assess reliability of neural network performance in response to unexpected execution contexts with the purpose of predicting potential safety-critical contexts.

Distributional shift assessment approaches such as OOD detection methods are usually demonstrated on MNIST, CIFAR or similar datasets and their applicability is seldomly shown for more complex datasets [Rabanser et al. 2018; Ren et al. 2019; Sehwag et al. 2021; Xiao et al. 2020]. While these benchmarks are useful, additional realistic datasets that reflect the problems of dealing with OOD inputs in real-world applications are required. With our research, we aim to fill this gap by evaluating state of the art OOD detection methods on autonomous driving camera data, while also demonstrating the influence of OOD data on the performance of a perception network error. As in-distribution data, we choose to select autonomous driving camera data recorded during nominal weather conditions, while out-of-distribution data is induced by adverse weather situations such a severe rain or fog. We evaluate data stemming from simulation with the CARLA Simulator [Dosovitskiy et al. 2017] as well as DrivingStereo, a real world driving dataset [Yang et al. 2019]. In order to assess the influence of distributional shift on the performance of a perceptual network, we employ a U-Net style neural network to estimate depth, trained with similar data as the OOD detection methods [Amini Alexander et al. 2019] Because a comprehensive outlier dataset is nearly unrealistic, we choose to only employ methods that detect outliers based only on in-distribution data. As a baseline method for OOD detection, we employ a variational autoencoder (VAE) trained on similar data as the depth estimation network and use a reconstruction error based out of distribution measure to estimate the distributional shift present in the data seen at test time. A similar approach termed Self-Oracle was recently introduced by Stocco et al. to address the problem of estimating the confidence of deep neural networks in response to unexpected execution contexts with the purpose of predicting potential safety-critical misbehaviours such as out of bound episodes or collisions [Stocco et al. 2020]. The authors showed that Self-Oracle was able predict $77 \%$ misbehaviours, outperforming the baseline input validation approach of DeepRoad by a factor of 3. Likelihood based approaches can also help exploit generative models like VAEs for OOD detection [Xiao et al. 2020]. However, recent research suggests that probabilistic generative models might assign greater likelihoods to particular types of OOD samples in specific situations, making likelihood threshold-based OOD detection problematic. However, several likelihood-based OOD detection strategies for deep generative models that circumvent this problem have recently been introduced [Ren et al. 2019; Xiao et al. 2020]. We choose to evaluate one new promising approach, termed Likelihood Regret, which has been shown to be an efficient OOD measure for VAEs [Xiao et al. 2020]. We also employ another promising recent approach based on generative modelling, which achieves state-ofthe-art performance called SSD. SSD uses self-supervised representation learning followed by a Mahalanobis distance based metric in the feature space, to calculate the OOD score [Sehwag et al. 2021]. We compare all 3 methods and evaluate them concurrently with the performance of the depth estimation network.

\subsection{Related work}

Distributional shift or out-of-distribution detection is the subject of various surveys and review articles [Bulusu et al. 2020; Chalapathy and Chawla 2019; Chandola et al. 2009]. There exist several approaches to the problem. Statistical approaches model the data based on its statistical properties and use this information to estimate whether a test instance comes from the same distribution or not. Classical approaches include Gaussian mixture models [Bishop 1994], hidden Markov models [Duda et al. 2001], and histograms [Taylor et al. 1985], which estimate the probability density function for the given data and apply thresholding for OOD detection. Although classic density estimators work reasonably well for low dimensional problems, they suffer notably from the curse of dimensionality. Recently, Rabanser et al. [Rabanser et al. 2018] proposed dimensionality reduction to combat this curse which is followed by statistical two-sample testing to evaluate the equivalence of the source distribution and target distribution for shift detection. A number of neural network based approaches have been proposed for OOD detection. Generative models such as autoencoders [Abati et al. 2019; Chalapathy et al. 2017; Chen et al. 2017; Chen and Konukoglu 2018; Huang et al. 2019; Pawlowski et al. 2018; Principi et al. 2017; Zhou and Paffenroth 2017; Zong et al. 2018] and variants such as the variational autoencoder [Chen et al. 2020; Cho 1992; Löhdefink et al. 2020; Park et al. 2018; Xu et al. 2018] are among the earliest and most widely used neural network based methods for OOD detection. During training, they learn a lower-dimensional latent space from which normal samples can be reconstructed, and then use some metric (e.g. the reconstruction error or likelihood) to identify an OOD sample during test time. For example, Stocco et al. [Stocco et al. 2020] propose an approach called Self-Oracle which employs an autoencoder based architectures to compute the reconstruction error followed by a probability distribution fitting to 
obtain a statistical model of the error. Based on this distribution, a threshold is estimated for anomaly detection. VAEs are probabilistic generative models that can evaluate the likelihood of input data and are able to indicate if they approximate the distribution of training data. However, recent studies [Choi et al. [n. d.]; Nalisnick et al. 2019] reveal that likelihoods obtained from current state-of-the-art deep probabilistic generative models sometimes fail to distinguish between training data and OOD input. To overcome the limitations of traditional likelihood based OOD detectors, Xiao et. al [Xiao et al. 2020] propose a simple metric called Likelihood Regret (LR) to detect OOD samples with VAEs. The Likelihood Regret of a single input can be interpreted as the log ratio between its likelihood obtained by the posterior distribution optimized individually for that input and the likelihood approximated by the trained VAE. Ren et. al [Ren et al. 2019] also propose a method based on likelihood ratios, which significantly outperforms the raw likelihood on OOD detection for deep generative models on image datasets. It uses a background model to correct for the background statistics and enhances the in-distribution specific features for OOD detection Serrà et. al [Serrà et al. 2019] propose another OOD score, that turns likelihood-based generative models into practical and effective OOD detectors, with performances comparable to, or even better than the state-of-the-art. They interpret this score as a likelihood-ratio, similar to Bayesian model comparison, because they use an estimate of input complexity to compensate standard negative log-likelihoods in order to produce an efficient and reliable OOD score. Generative adversarial networks (GAN) can also be exploited for OOD detection. GAN-based method allows to learn generative models that can generate realistic images [Denton et al. 2015; Donahue et al. 2016; Goodfellow et al. 2014]. Schlegl et. al [Schlegl et al. 2017] use a GAN-based unsupervised learning model named AnoGan to identify anomalies in medical imaging data. The authors propose to use a combination of the 12-norm and a discrimination loss between a query image and its closest reconstruction match as an anomaly score. Based on this approach, Deecke et al. [Deecke et al. 2019] proposed a similar method termed Attribute-Decomposed GAN (ADGAN) that, given a sample under consideration, searches for a good representation of that sample in the latent space of the generator. If such a representation is not found, the sample is considered anomalous. Recently, Schlegl et al. [Schlegl et al. 2019] proposed a GAN-based fast unsupervised anomaly detection (f-AnoGAN), improving upon AnoGAN, by using an encoder instead of an iterative optimization procedure to enable a fast mapping from images to the learned latent representation in a single step during inference. This makes the proposed technique suitable for real-time anomaly detection applications. Sehwag et al. [Sehwag et al. 2021] introduced another generative method for OOD detection called SSD, which is an unsupervised framework for outlier detection based on unlabeled in-distribution data. SSD uses self-supervised representation learning to develop a rich understanding of key semantics in in-distribution data, followed by a Mahalanobis distance based outlier detection to identify outliers that are far away in the feature space.

\section{METHODS}

\subsection{Distributional Shift Detection Methods}

The first step in our process consists of training the different distributional shift detection models under nominal weather conditions. At the end of this step, for each approach, a metric distribution for the training data can be computed. In case of the reconstruction error based VAE, a reconstruction error can be computed for all images in the training set, yielding an error distribution characterizing the training dataset. When facing an in-distribution test sample, a well-trained VAE should show similar reconstruction errors as present in the training dataset, while yielding a considerably larger reconstruction error for an OOD test sample. [Stocco et al. 2020] For a more detailed description of the model and methods, please see [Stocco et al. 2020]. Likelihood regret (LR) estimates the log likelihood improvement of the model that maximizes the likelihood of a specific sample over the model configuration that maximizes the likelihood across all training samples. For more details, please refer to [Xiao et al. 2020]. When facing an in-distribution test sample, a well-trained VAE should show little change in likelihood from swapping the present model configuration with the best one for the single test sample, yielding a low LR score. While, in contrast, the trained model configuration is considerably less likely to be near the optimal for an OOD test sample, and hence the LR should be larger [Xiao et al. 2020]. SSD consists of two steps: First, a feature extractor (PixelCNN) is trained using unsupervised contrastive representation learning. Next, an outlier score is computed, based on the Mahalanobis distance metric in the feature space. When confronted with an in-distribution test sample, a well-trained PixelCNN should yield similar features as present in the training dataset, resulting in a distance metric comparable to the training distribution, while yielding a considerably larger distance for an OOD test sample [Sehwag et al. 2021]. Please refer to [Sehwag et al. 2021] for a detailed description of methods.

\subsection{Depth prediction}

We use a monocular depth estimate methodology to assess the impact of out of distribution data on perception performance. Endto-end monocular depth estimation is a key topic in computer vision which entails learning a representation of depth directly from an RGB camera image. We train a U-Net type neural network for depth inference with similar data that we train our OOD detection models, as described below. [Ronneberger et al. 2015]. We evaluate the model performance in terms of RMSE across all pixels. We expect that depth estimation for in-distribution data is better than for OOD data.

\subsection{Datasets}

With the selection of training and evaluation datasets for OOD detection models and depth estimation network we want to simulate the situation of a car OEM, who wants to assess the reliability of their perceptual network models with an OOD metric, but who does not have access to the complete training data that was used for training a specific neural network for perception. For evaluation, we use simulation data from the CARLA simulator [Dosovitskiy 
et al. 2017] as well as real world driving data from the DrivingStereo dataset [Yang et al. 2019]. In the simulator, for generating the depth estimation network training data, we record camera data on unique routes from each CARLA map available (8 different maps) with nominal sunny weather conditions during 4 daylight regimes, morning, noon, afternoon and early evening. Similarly, for generating training data for DSD methods, we also record camera data on unique different routes from each CARLA map available under the same conditions as above. For testing depth perception network and DSD methods on OOD data, we generate camera data on the same maps and routes with gradually increasing fog by manipulating fog density parameters. We simulate 5 different levels of fog as shown in Figure 1. All together, the depth network and DSD training set consist out of 32.000 images, and the test set consists of 160.000 images.

For training and testing with real world data, we use the Driving Stereo dataset [Yang et al. 2019]. We use a unique subset of images from each sequence with nominal weather conditions (Figure 2 (a)) randomly selected for training of depth estimation model, DSD model training and DSD model evaluation. For training of depth estimation model, we select random images from each sequence. Similarly, for DSD model training, we select another set of unique random images from each sequence. For evaluation of our DSD methods, we use foggy and rainy scenes as out-of-distribution data, shown in Figure 2 (b) and (c) respectively. All together, the depth network and DSD training set consist out of 6.000 images, and the test set consists of 500 rainy and 500 foggy images.

\section{RESULTS}

\subsection{Simulated data}

The evaluation of depth prediction error and selected OOD metrics on CARLA simulation data [Dosovitskiy et al. 2017] is shown in Figure 3 (left column). The time-series starts with a baseline lap under nominal weather conditions, included in DSD model training, but not in depth estimation model training, followed by 5 laps with gradually increasing fog, not included in DSD model nor depth estimation model training. The error of the depth estimation neural network starts with low values under nominal weather conditions, although the network has not seen those specific images during training, but has been trained with similar data, as described above. With increasing fog, the depth prediction error increases and plateaus at around $60 \%$ percent fog intensity. SelfOracle is not able to differentiate clearly between images it has been trained on and images with low fog intensity with regard to the reconstruction error. With increasing fog however, reconstruction error also increases showing signs of correlating with the depth prediction error with $r=0.74$ (Pearson correlation coefficient). The Likelihood-Regret (LR) based method shows low scores for baseline images it has been trained with and displays a strong increase in likelihood-regret values when applied to images with increasing fog, without being able to differentiate much between different levels of fog, with $r=0.75$. SSD assigns low scores to baseline data with which it has been trained, with scores increasing nearly linearly with increasing fog intensities. With $r=0.77$, SSD scores show a strong correlation with the depth estimation error. To complement time-series analysis, we investigated depth estimation and OOD metric distributions on CARLA simulation data, as shown in Figure 3 (right column). The histograms for depth estimation and each DSD approach display metrics for DSD training data in blue, metrics for depth estimation training data in red, metrics for foggy images, labeled as OOD data, in lavender. Depth estimation error (RMSE) shows a similar distribution for depth estimation training data and DSD training data, both representing nominal weather conditions. The error for foggy images is clearly higher overall, with error values for low fog conditions partly overlapping with error values for nominal weather conditions. Self-Oracle shows a large intersection between all three distributions, indicating that the training, depth estimation training, and OOD data are not clearly separated by the metric. LR shows a pronounced difference between the training and depth estimation training metric distributions, revealing the method's sensitivity to the training data. However, metrics for OOD data are well separated from both the distributions. SSD shows a pronounced overlap between metric distributions for training and depth estimation training data, while the distribution of OOD data metrics is mostly separated. The small intersection of the OOD data corresponds to the low fog intensity (primarily 20\%) showing similar SSD scores as training data, which can be observed from the time series analysis in Figure 3 (left column).

\subsection{Real-world data}

The evaluation of depth prediction error and DSD methods on real world data [Yang et al. 2019] is shown in Figure. 4 (left column). The time-series starts with a baseline sequence under nominal weather conditions, followed by a sequence with foggy and rainy weather. Depth prediction error starts with low values under nominal weather conditions and increases to high values when fog or rain is encountered. Self-Oracle is unable to distinguish between images it has been trained on and foggy images as both are assigned similar reconstruction errors. With $r=0.068$, Self-Oracle demonstrates no correlation with depth prediction performance. For rainy images, reconstruction error values decrease, showing a negative correlation with depth estimation error, with $r=-0.49$. LR shows low scores for baseline images it has been trained with, while exhibiting a strong increase in likelihood-regret values when applied to images with fog (correlation with depth estimation error, $r=$ $0.4)$ and rain $(r=0.67)$. SSD assigns low scores to the baseline data with which it has been trained, with scores increasing for foggy and rainy weather. SSD scores show a modest association with the depth estimation performance, with $r=0.53$ and 0.71 for fog and rain, respectively. Metric distributions on real world data are shown in Figure 4 (right column). The histograms for the depth estimation and each DSD method display metrics for DSD training data in blue, depth estimation training data, on which the depth estimation network has been trained on, in red, metrics for foggy images, labeled as OOD data, in lavender and metrics for rainy images, in light blue. Depth estimation error (RMSE) shows a similar distribution for depth estimation training data and DSD training data, both representing nominal weather conditions, with DSD training data error values slightly higher overall. The error for foggy and rainy images is clearly higher overall, with higher error values for rainy conditions, in which fog is also partly present. Self-Oracle shows a considerable overlap between training, depth estimation training, 


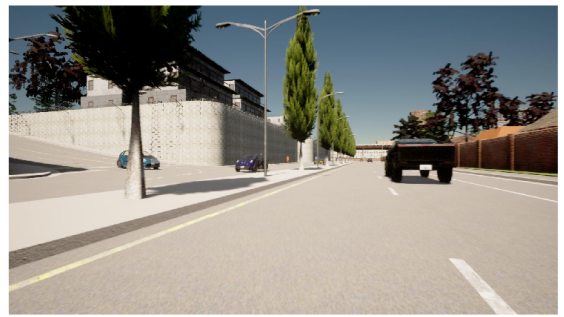

(a) Normal

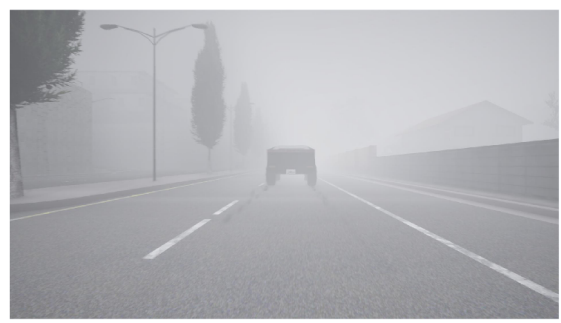

(d) $60 \%$ Fog

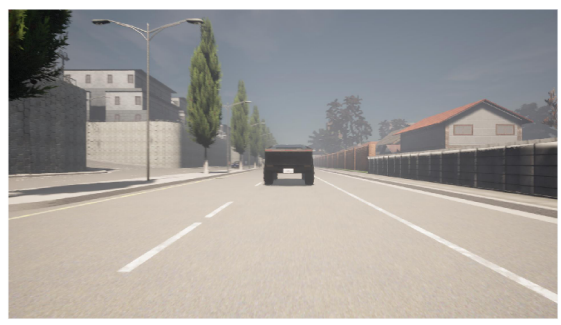

(b) $20 \%$ Fog

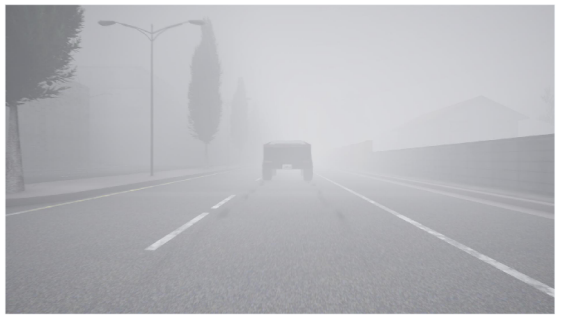

(e) $80 \%$ Fog

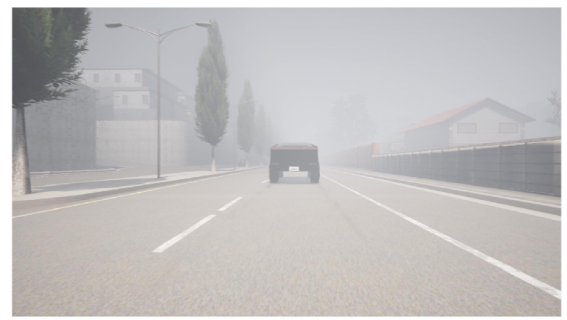

(c) $40 \%$ Fog

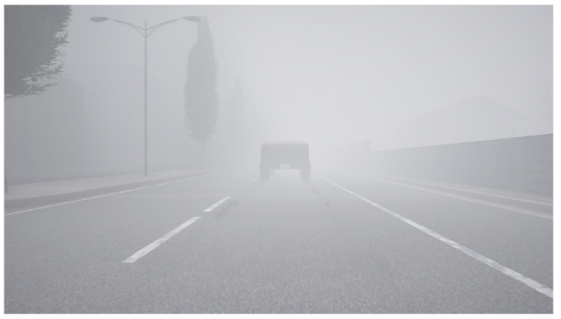

(f) $100 \%$ Fog

Figure 1: Example images from Carla dataset [Dosovitskiy et al. 2017]. (a) depicts normal weather used for DSD and depth estimation network training, while (b)-(f) depict foggy weather with $20,40,60,80,100$ percent fog intensity, respectively used for OOD testing.

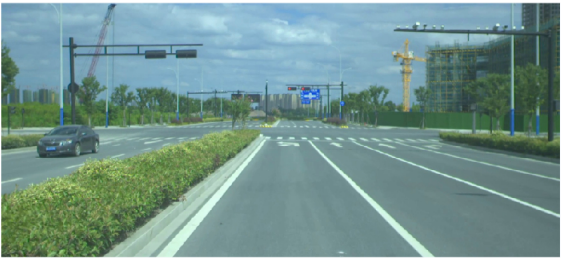

(a) Normal

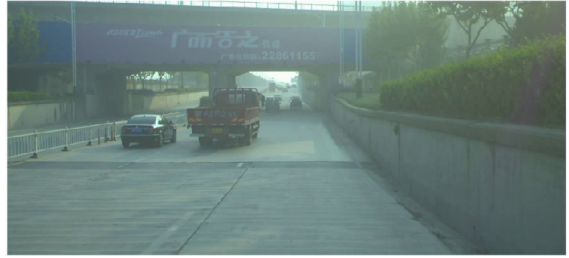

(b) Foggy

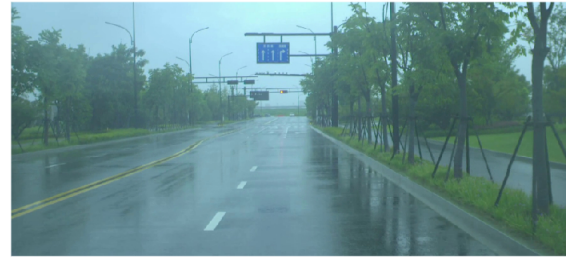

(c) Rainy

Figure 2: Example images from Driving Stereo dataset [Yang et al. 2019]. (a) depicts normal weather for DSD and depth estimation network training, while (b) and (c) depict foggy and rainy weather for OOD testing, respectively.

and fog OOD metric distribution, indicating that they have similar reconstruction errors, while displaying lower metric values for rainy data. The training and depth estimation training data metric distributions are clearly separated in LR, revealing the method's sensitivity to the training data; however, scores for OOD data (both fog and rain) are well separated from both the distributions. SSD has a large overlap between metric distribution for training and depth estimation training data, while keeping OOD data metric (fog and rain) separated from both the distribution.

\section{DISCUSSION AND CONCLUSION}

With this study, we evaluated three state of the art distributional shift detection methods on simulated and real autonomous driving camera data, while also demonstrating the influence of OOD data on the performance of a depth estimation neural network, which is typically part of an autonomous driving perception system. We show that Distributional Shift can be detected and quantified in simulated and real-world data with selected methods. However, not all methods perform equally well in all evaluated scenarios. In the original study, Self-Oracle was able to anticipate many potentially safety-critical misbehaviours by several seconds, such as collisions or out of bound episodes, outperforming the baseline validator of DeepRoad [Stocco et al. 2020]. For anticipation, SelfOracle exploits the reconstruction error distribution of the training data and compares it with test time reconstruction error. We find that VAEs often obtain similar reconstruction errors on OOD data, confirming the results of Xiao et al. [Xiao et al. 2020], who also obtained surprisingly good reconstruction quality on OOD data. We show that Self-Oracle test time reconstruction error distribution often completely overlaps with the training distribution broadly, while the perceptual task network (depth estimation) additionally shows degraded performance on test images. Our results show the 

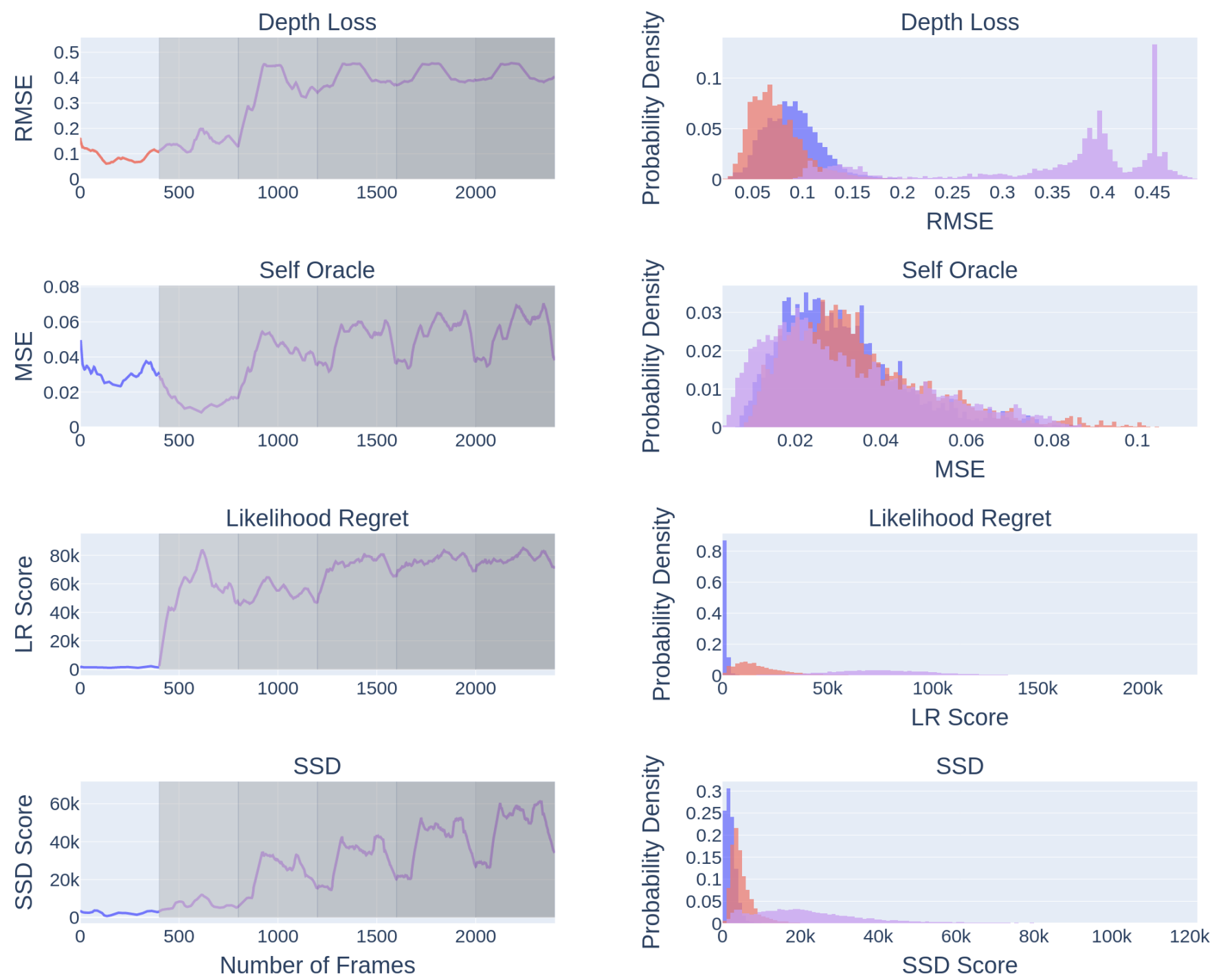

\section{-Fog $=$ DSD Training Data $=$ Depth Estimation Training Data $"$ Out of Distribution Data Fog}

Figure 3: Left column: Evaluation of depth estimation performance and OOD metrics on simulation data with fog test data. The first 500 frames depict a lap around a CARLA town under nominal weather conditions used in DSD training, whereas the following frames depict the same lap with a gradual increase in fog intensity from $20 \%$ to $100 \%$ from lap to lap, indicated by different shades of grey. The error of the depth estimation neural network starts with low values under nominal weather conditions and with increasing fog, the depth prediction error increases reaches a plateau. Self-Oracle is unable to differentiate fog from baseline at low intensities but shows a good correlation with depth error at high intensities. Likelihood regret (LR) rises to a high value as soon as fog is encountered and correlates with depth error at higher intensities. SSD gradually increases with fog intensity and shows a linear relation with depth error. Values displayed are smoothed with a moving average filter with a window size of 50. Right column: Depth estimation error and OOD metric distributions for DSD training data (blue), depth estimation training data (red) and fog test data (lavender) with simulation data. Self-Oracle shows an overlap between all the three distribution, indicating it is not able to differentiate clearly between in distribution and out of distribution data. LR separates the OOD data with a wide margin from the training data. LR training score distribution overlaps partly with depth estimation training data score distribution, while depth estimation data training score distribution overlaps marginally with OOD data. SSD shows a strong overlap between training and depth estimation training data, with marginal overlap with the OOD data, likely stemming from images with low fog intensities, as shown in the timeseries analysis. 

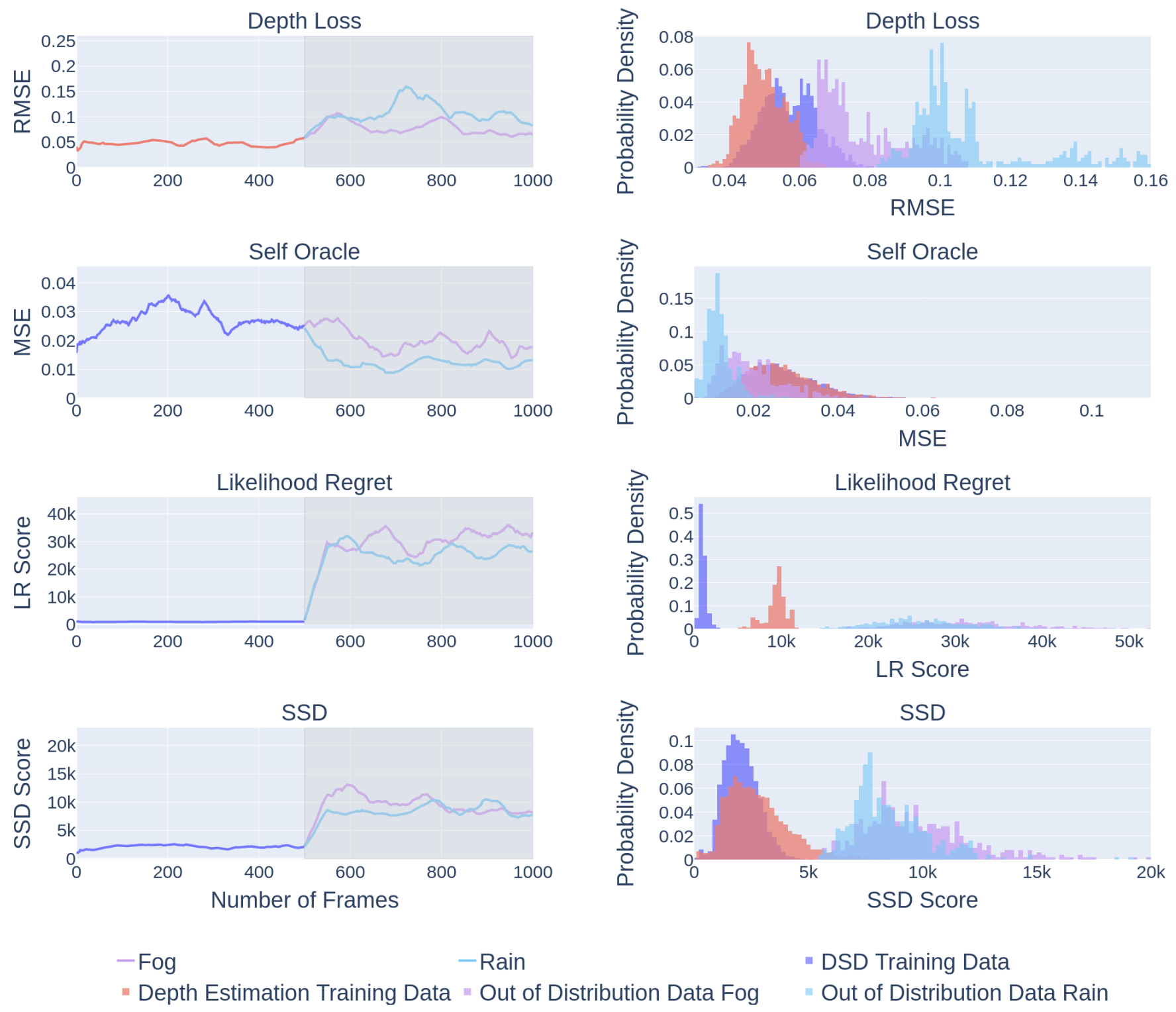

Figure 4: Left column: Evaluation of depth estimation error and OOD metrics on DrivingStereo dataset with foggy and rainy test data. The first 500 frames depict a sequence of images under nominal weather conditions used in DSD training, whereas the following $\mathbf{5 0 0}$ frames depict images from foggy sequences in lavender and rainy sequences in light blue. The error of the depth estimation neural network starts with low values under nominal weather conditions and with fog and rain, the depth prediction error increases above baseline levels. Self-Oracle is unable to differentiate fog and rain from baseline. Likelihood regret and SSD score rise clearly above baseline levels as soon as fog and rain is encountered. Values displayed are smoothed with a moving average filter with a window size of 50. Right column: Depth estimation error and OOD metric distributions for DSD training data (blue), depth estimation training data (red) and foggy test data (lavender) and rainy test data (light blue) with DrivingStereo dataset. Self-Oracle shows an overlap between all the three distribution, indicating it is not able to differentiate clearly between in distribution and out of distribution data. LR separates the OOD data with a wide margin from the training data. LR training score distribution does not overlap with the score distribution for foggy and rainy images, while depth estimation data training score distribution does not overlap with OOD data scores. SSD shows a strong overlap between training and depth estimation training data scores, with marginal overlap with the OOD data scores. 
Table 1: Correlation coefficients of depth estimation error and DSD metrics.

\begin{tabular}{||cccc||}
\hline DSD Method & Simulated Fog & DrivingStereo Fog & DrivingStereo Rain \\
\hline \hline Self-Oracle & 0.74 & 0.06 & -0.49 \\
\hline Likelihood Regret & 0.75 & 0.4 & 0.67 \\
\hline SSD & $\mathbf{0 . 7 7}$ & $\mathbf{0 . 5 3}$ & $\mathbf{0 . 7 1}$ \\
\hline
\end{tabular}

limits of this method for OOD detection and its applicability for the prediction of safety-critical contexts. Across all scenarios, LR and SSD approaches consistently differentiate between training data and out of distribution data (see Table 1). Both metrics show a high correlation with perception performance across contexts with SSD persistently displaying the highest correlation coefficients. Both methods do well in separating OOD data from training data, with LR consistently showing a wider margin between both distributions. While LR is very sensitive to the training data and predicts higher scores for similar data, which has not been used in training, SSD scores only marginally separate method training and unseen but similar data. As LR performs well in OOD detection and the LR score is correlated with performance of the neural network on the depth estimation task, but as it is also very sensitive to the training data distribution, this method could be a promising input signal for an early reliability warning monitoring system within the autonomous driving system. SSD performs well in OOD detection, the SSD score is highly correlated with perceptual task performance and also displays similar score distributions for similar input data. This method could be a robust reliability quantifier in a safety monitoring system within the autonomous driving system. Compared with LR, SSD metric computation is faster, because no model fitting is needed during metric inference. Our encouraging results in distributional shift detection and their applicability to neural network prediction reliability assessment can aid in improving the safety of autonomous driving systems. Future work will focus on improving OOD detection by e.g. exploiting Vision Transformer based architectures and sophisticated self-supervised training strategies [Fort et al. 2021; Li et al. 2021] for image representation along with integrating different reliability measures [Chen et al. 2021] into a system safety monitor. White-box reliability assessment approaches such as fast uncertainty estimation based on deep ensembles [Valdenegro-Toro 2019] or deep evidential learning, as introduced by Amini et al. [Amini Alexander et al. 2019], who present a novel training method for non-Bayesian neural networks to estimate a continuous target as well as its associated evidence, could complement black-box assessment methods.

\section{REFERENCES}

Davide Abati, Angelo Porrello, Simone Calderara, and Rita Cucchiara. 2019. Latent space autoregression for novelty detection. In Proceedings of the IEEE Computer Society Conference on Computer Vision and Pattern Recognition. https://doi.org/10. 1109/CVPR.2019.00057

Amini Alexander, Schwarting Wilko, Soleimany Ava, and Rus Daiela. 2019. Deep Evidential Regression. (2019).

C. M. Bishop. 1994. Novelty detection and neural network validation. IEE Proceedings: Vision, Image and Signal Processing (1994). https://doi.org/10.1049/ip-vis:19941330

Saikiran Bulusu, Bhavya Kailkhura, Bo Li, Pramod K. Varshney, and Dawn Song. 2020. Anomalous Example Detection in Deep Learning: A Survey. IEEE Access 8 (2020) https://doi.org/10.1109/ACCESS.2020.3010274

Raghavendra Chalapathy and Sanjay Chawla. 2019. Deep learning for anomaly detection: A survey.
Raghavendra Chalapathy, Aditya Krishna Menon, and Sanjay Chawla. 2017. Robust, Deep and Inductive Anomaly Detection. In Lecture Notes in Computer Science (including subseries Lecture Notes in Artificial Intelligence and Lecture Notes in Bioinformatics). https://doi.org/10.1007/978-3-319-71249-9\{\}3

Varun Chandola, Arindam Banerjee, and Vipin Kumar. 2009. Anomaly detection: A survey. https://doi.org/10.1145/1541880.1541882

Jinghui Chen, Saket Sathe, Charu Aggarwal, and Deepak Turaga. 2017. Outlier detection with autoencoder ensembles. In Proceedings of the 17th SIAM International Conference on Data Mining, SDM 2017. https://doi.org/10.1137/1.9781611974973.11

Xiaoran Chen and Ender Konukoglu. 2018. Unsupervised detection of lesions in brain MRI using constrained adversarial auto-encoders. https://doi.org/10.3929/ethz-b000321650

Xiaoran Chen, Suhang You, Kerem Can Tezcan, and Ender Konukoglu. 2020. Unsupervised Lesion Detection via Image Restoration with a Normative Prior.

Yiqiang Chen, Feng Liu, and Ke Pei. 2021. Cross-Modal Matching CNN for Autonomous Driving Sensor Data Monitoring. In Proceedings of the IEEE/CVF International Conference on Computer Vision (ICCV) Workshops. 3110-3119.

An; Cho. 1992. Variational Autoencoder based Anomaly Detection using Reconstruction Probability. Fournal of The Electrochemical Society (1992).

Hyunsun Choi, Eric Jang, and Alexander A Alemi. [n. d.]. WAIC, but Why? Generative Ensembles for Robust Anomaly Detection. Technical Report.

Lucas Deecke, Robert Vandermeulen, Lukas Ruff, Stephan Mandt, and Marius Kloft. 2019. Image anomaly detection with generative adversarial networks. In Lecture Notes in Computer Science (including subseries Lecture Notes in Artificial Intelligence and Lecture Notes in Bioinformatics), Vol. 11051 LNAI. https://doi.org/10.1007/9783-030-10925-7\{\}1

Emily Denton, Soumith Chintala, Arthur Szlam, and Rob Fergus. 2015. Deep Generative Image Models using a Laplacian Pyramid of Adversarial Networks. Technical Report.

Jeff Donahue, Philipp Krähenbühl, and Trevor Darrell. 2016. Adversarial Feature Learning. (2016)

Alexey Dosovitskiy, German Ros, Felipe Codevilla, Antonio López, and Vladlen Koltun. 2017. CARLA: An Open Urban Driving Simulator. Technical Report.

R O Duda, P E Hart, and D G Stork. 2001. Pattern classification. New York: fohn Wiley, Section (2001).

Stanislav Fort, Jie Ren, and Balaji Lakshminarayanan. 2021. Exploring the Limits of Out-of-Distribution Detection. arXiv:2106.03004 [cs.LG]

Ian J. Goodfellow, Jean Pouget-Abadie, Mehdi Mirza, Bing Xu, David Warde-Farley, Sherjil Ozair, Aaron Courville, and Yoshua Bengio. 2014. Generative adversarial nets. In Advances in Neural Information Processing Systems, Vol. 3. https://doi.org/ 10.3156/jsoft.29.5\{_\}177\{_\}2

Manuel Gunther, Steve Cruz, Ethan M. Rudd, and Terrance E. Boult. 2017. Toward Open-Set Face Recognition. In IEEE Computer Society Conference on Computer Vision and Pattern Recognition Workshops, Vol. 2017-July. https://doi.org/10.1109/CVPRW. 2017.85

Chaoqing Huang, Jinkun Cao, Fei Ye, Maosen Li, Ya Zhang, and Cewu Lu. 2019. Inversetransform autoencoder for anomaly detection.

Quinonero-Candela Joaquin, Masashi Sugiyama, Anton Schwaighofer, and Neil D Lawrence. 2008. Dataset Shift in Machine Learning (Neural Information Processing).

Chunyuan Li, Jianwei Yang, Pengchuan Zhang, Mei Gao, Bin Xiao, Xiyang Dai, Lu Yuan, and Jianfeng Gao. 2021. Efficient Self-supervised Vision Transformers for Representation Learning. arXiv:2106.09785 [cs.CV]

Jonas Löhdefink, Justin Fehrling, Marvin Klingner, Fabian Hüger, Peter Schlicht, Nico M. Schmidt, and Tim Fingscheidt. 2020. Self-Supervised Domain Mismatch Estimation for Autonomous Perception. CoRR abs/2006.08613 (2020). https://arxiv.org/abs/ 2006.08613

Iacopo Masi, Yue Wu, Tal Hassner, and Prem Natarajan. 2019. Deep Face Recognition: A Survey. In Proceedings - 31st Conference on Graphics, Patterns and Images, SIBGRAPI 2018. https://doi.org/10.1109/SIBGRAPI.2018.00067

Eric Nalisnick, Akihiro Matsukawa, Yee Whye Teh, Dilan Gorur, and Balaji Lakshminarayanan. 2019. Do deep generative models know what they don't know?. In 7th International Conference on Learning Representations, ICLR 2019.

Daehyung Park, Yuuna Hoshi, and Charles C. Kemp. 2018. A Multimodal Anomaly Detector for Robot-Assisted Feeding Using an LSTM-Based Variational Autoencoder. IEEE Robotics and Automation Letters (2018). https://doi.org/10.1109/LRA.2018. 2801475 
Nick Pawlowski, Matthew C H Lee, Martin Rajchl, Steven Mcdonagh, Enzo Ferrante, Konstantinos Kamnitsas, Sam Cooke, Susan Stevenson, Aneesh Khetani, Tom Newman, Fred Zeiler, Richard Digby, Jonathan P Coles, Daniel Rueckert, David K Menon, Virginia F J Newcombe, and Ben Glocker. 2018. Unsupervised Lesion Detection in Brain CT using Bayesian Convolutional Autoencoders. Technical Report.

Emanuele Principi, Fabio Vesperini, Stefano Squartini, and Francesco Piazza. 2017. Acoustic novelty detection with adversarial autoencoders. In Proceedings of the International foint Conference on Neural Networks. https://doi.org/10.1109/IJCNN. 2017.7966273

Stephan Rabanser, Stephan Günnemann, and Zachary C. Lipton. 2018. Failing loudly: An empirical study of methods for detecting dataset shift.

Manikandasriram Srinivasan Ramanagopal, Cyrus Anderson, Ram Vasudevan, and Matthew Johnson-Roberson. 2018. Failing to Learn: Autonomously Identifying Perception Failures for Self-Driving Cars. IEEE Robotics and Automation Letters 3, 4 (2018). https://doi.org/10.1109/LRA.2018.2857402

Jie Ren, Peter J. Liu, Emily Fertig, Jasper Snoek, Ryan Poplin, Mark A. DePristo, Joshua V. Dillon, and Balaji Lakshminarayanan. 2019. Likelihood ratios for out-of-distribution detection.

Olaf Ronneberger, Philipp Fischer, and Thomas Brox. 2015. U-net: Convolutional networks for biomedical image segmentation. In Lecture Notes in Computer Science (including subseries Lecture Notes in Artificial Intelligence and Lecture Notes in Bioinformatics), Vol. 9351. https://doi.org/10.1007/978-3-319-24574-4\{\}28

Thomas Schlegl, Philipp Seeböck, Sebastian M. Waldstein, Georg Langs, and Ursula Schmidt-Erfurth. 2019. f-AnoGAN: Fast unsupervised anomaly detection with generative adversarial networks. Medical Image Analysis 54 (2019). https://doi org/10.1016/j.media.2019.01.010

Thomas Schlegl, Philipp Seeböck, Sebastian M. Waldstein, Ursula Schmidt-Erfurth, and Georg Langs. 2017. Unsupervised anomaly detection with generative adversarial networks to guide marker discovery. In Lecture Notes in Computer Science (including subseries Lecture Notes in Artificial Intelligence and Lecture Notes in Bioinformatics), Vol. 10265 LNCS. https://doi.org/10.1007/978-3-319-59050-9\{_\}12

Vikash Sehwag, Mung Chiang, and Prateek Mittal. 2021. SSD: A UNIFIED FRAMEWORK FOR SELF-SUPERVISED OUTLIER DETECTION. Technical Report. https://github.
com/inspire-group/SSD

Joan Serrà, Davidalvarez David Davidalvarez, Vicenç Gómez, Olga Slizovskaia, José F N U Nez, and Jordi Luque. 2019. INPUT COMPLEXITY AND OUT-OFDISTRIBUTION DETECTION WITH LIKELIHOOD-BASED GENERATIVE MODELS. Technical Report.

Andrea Stocco, Michael Weiss, Marco Calzana, and Paolo Tonella. 2020. Misbehaviour prediction for autonomous driving systems. In Proceedings - International Conference on Software Engineering. https://doi.org/10.1145/3377811.3380353

C. C. Taylor, Luc Devroye, and Laszlo Gyorfi. 1985. Nonparametric Density Estimation: The L 1 View. Journal of the Royal Statistical Society. Series A (General) (1985). https://doi.org/10.2307/2981908

Matias Valdenegro-Toro. 2019. Deep Sub-Ensembles for Fast Uncertainty Estimation in Image Classification.

Riccardo Volpi and Vittorio Murino. 2019. Addressing Model Vulnerability to Distributional Shifts over Image Transformation Sets. Technical Report.

Zhisheng Xiao, Qing Yan, and Yali Amit. 2020. Likelihood Regret: An Out-of-Distribution Detection Score For Variational Auto-encoder. Technical Report.

Haowen Xu, Wenxiao Chen, Nengwen Zhao, Zeyan Li, Jiahao Bu, Zhihan Li, Ying Liu, Youjian Zhao, Dan Pei, Yang Feng, Jie Chen, Zhaogang Wang, and Honglin Oiao. 2018. Unsupervised Anomaly Detection via Variational Auto-Encoder for Seasonal KPIs in Web Applications. In The Web Conference 2018 - Proceedings of the World Wide Web Conference, WWW 2018. https://doi.org/10.1145/3178876.3185996

Guorun Yang, Xiao Song, Chaoqin Huang, Zhidong Deng, Jianping Shi, and Bolei Zhou. 2019. DrivingStereo: A Large-Scale Dataset for Stereo Matching in Autonomous Driving Scenarios. Technical Report.

Chong Zhou and Randy C. Paffenroth. 2017. Anomaly detection with robust deep autoencoders. In Proceedings of the ACM SIGKDD International Conference on Knowledge Discovery and Data Mining. https://doi.org/10.1145/3097983.3098052

Bo Zong, Qi Song, Martin Renqiang Min, Wei Cheng, Cristian Lumezanu, Daeki Cho, and Haifeng Chen. 2018. Deep autoencoding Gaussian mixture model for unsupervised anomaly detection. In 6 th International Conference on Learning Representations, ICLR 2018 - Conference Track Proceedings. 